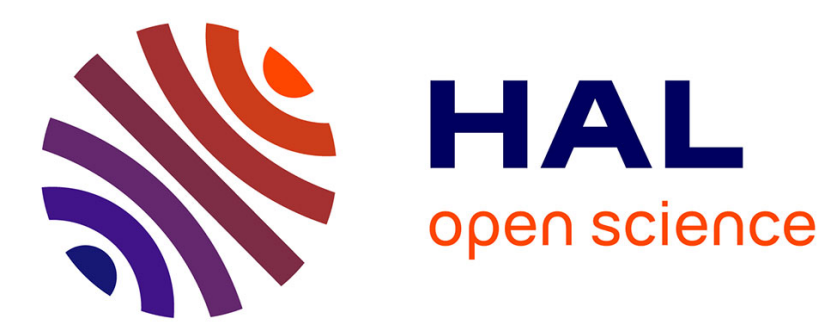

\title{
A 2-D anatomic breast ductal computer phantom for ultrasonic imaging
}

\author{
Emilie Franceschini, Serge Mensah, D. Amy, Jean-Pierre Lefebvre
}

\section{To cite this version:}

Emilie Franceschini, Serge Mensah, D. Amy, Jean-Pierre Lefebvre. A 2-D anatomic breast ductal computer phantom for ultrasonic imaging. IEEE Transactions on Ultrasonics, Ferroelectrics and Frequency Control, 2006, 53 (7), pp.1281-1288. hal-00089010

\section{HAL Id: hal-00089010 https://hal.science/hal-00089010}

Submitted on 18 Dec 2008

HAL is a multi-disciplinary open access archive for the deposit and dissemination of scientific research documents, whether they are published or not. The documents may come from teaching and research institutions in France or abroad, or from public or private research centers.
L'archive ouverte pluridisciplinaire $\mathbf{H A L}$, est destinée au dépôt et à la diffusion de documents scientifiques de niveau recherche, publiés ou non, émanant des établissements d'enseignement et de recherche français ou étrangers, des laboratoires publics ou privés. 


\title{
A 2-D anatomic breast ductal computer phantom for ultrasonic imaging *
}

\author{
Emilie Franceschini ${ }^{1}$, Serge Mensah ${ }^{1}$, \\ Dominique Amy ${ }^{2}$, Jean-Pierre Lefebvre ${ }^{1}$ \\ ${ }^{1}$ Laboratoire de Mécanique et d'Acoustique - CNRS \\ 31 Chemin Joseph Aiguier, \\ 13402 Marseille Cedex 20 - France \\ ${ }^{2}$ University of Medecine Montpellier-Nîmes, \\ UFR Kennedy, 30908 Nîmes, Cedex 2, France.
}

May 26, 2006

\begin{abstract}
Most breast cancers $(85 \%)$ originate from the epithelium and develop first in the ductolobular structures. In screening procedures, the mammary epithelium should therefore be investigated first by performing of an anatomically guided examination. For this purpose (mass screening, surgical guidance), we developed a two-dimensional anatomic phantom corresponding to an axial cross-section of the ductolobular structures, which makes it possible to better understand the interactions between the breast composition and ultrasound. The various constitutive tissues were modeled as a random inhomogeneous continuum with density and sound speed fluctuations. Ultrasonic pulse propagation through the breast computer phantom was simulated using a finite element time domain method (the phantom can be used with other propagation codes). The simulated Ductal Echographic image is compared with the Ductal Tomographic (DT) reconstruction. The preliminary results obtained show that the DT method is more satisfactory in terms of both the contrast and the resolution.
\end{abstract}

Key Words : Breast model, Ductal Tomography, Ductal Echography, FEM, nearfield.

${ }^{*}$ Version of the preprint submitted to IEEE Transactions on Ultrasonics, Ferroelectrics and Frequency Control 


\section{Introduction}

The breast consists of soft tissue of three kinds. First there is a variable volume of fatty tissue in which 15 to 20 lobes radiate from the nipple in a daisy-like arrangement. Within each lobe, one can find many grape-like hollow structures called epithelial or ductolobular structures [1] [2]. The internal walls of the ductolobular structures are lined with an extremely thin 50-micron one to two cell-thick layer of epithelial tissue. Figure 1 shows the successive layers composing the breast: the skin, the superficial fatty layer, the lobes and the ductolobular structures. Most breast cancers (85\%) originate from the epithelium and develop first in the ductolobular structures [3]. Observations on these structures therefore provide direct means of detecting any alterations induced by breast diseases as soon as they become perceptible. Unfortunately, conventional means of diagnosis do not show epithelial structures and this often makes them inefficient.

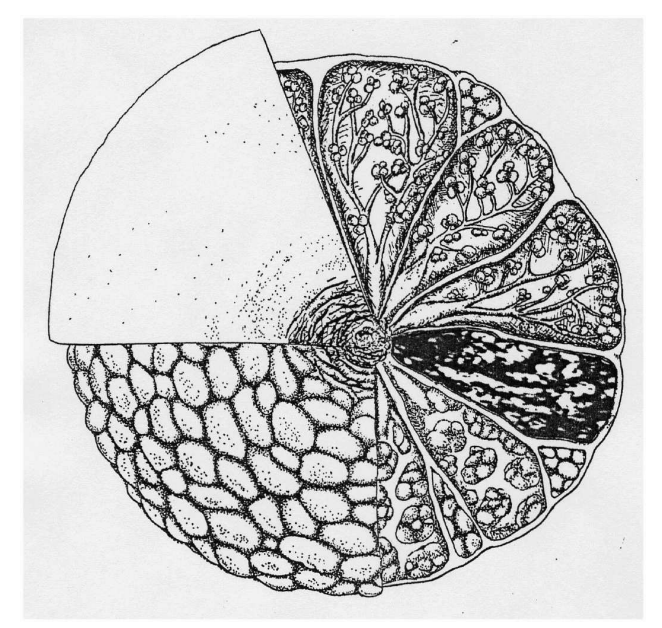

Figure 1: Clock-face arrangement of the ductolobular structures [1].

Three noninvasive methods of investigation are commonly used for mammary exploration:

- X-ray mammography is the "gold standard" in breast examination. Its widespread use was due to the fact that it is practical, simple to use, operator independent and gives quick global views. However, X-ray mammography does not visualize epithelium, which is radio transparent. The diseases detected are restricted to the affected tissues that have had a period of development long enough to produce a sufficiently dense connective reaction to become mammographically distinguishable.

- Magnetic Resonance Imaging (MRI) is a technique that allows the visualization of the three breast tissue constituents (connective tissue, fat and the ductolobular structures). MRI is being increasingly employed, especially for breast examinations on young women. However, today, the resolution is still insufficient [2] and this method is limited by economic and technical factors.

- Echography is well adapted to soft tissue examinations but its utilization to date has not been fully appreciated as a clinical means of routine screening. 
The role of echography in breast investigation has been limited by different factors. Analysis and interpretation of echographic data present several difficulties, the results are not easily reproducible, and its quality is highly dependent on the operator. Moreover, in conventional echography, transductal scans are performed perpendicularly to the ductal course. This mode of scanning does not match the radial arrangement of the breast anatomy. Cancers can be detected only when they show a sufficiently high contrast and the abnormalities have a sufficiently large volume to be perceptible whatever the sweeping orientation ( $5 \mathrm{~mm}$ size lesions). The lack of anatomic guidance in breast ultrasound has been one of the causes for its lack of progress in clinical breast screening.

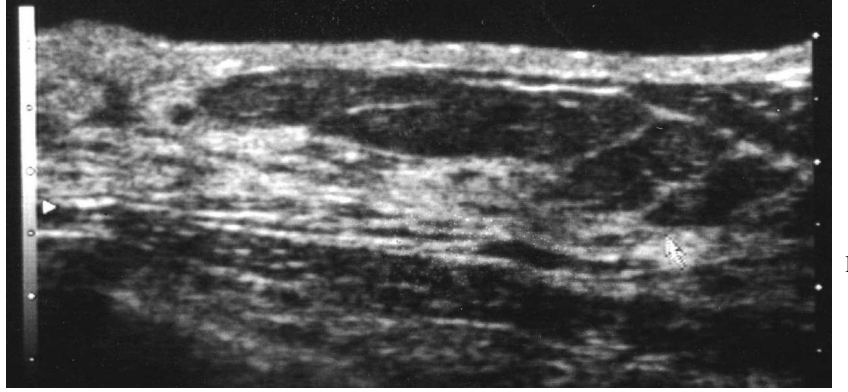

(a)

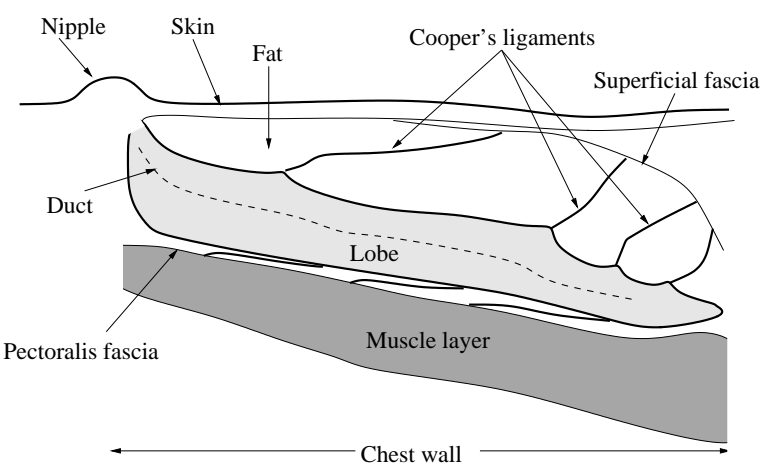

(b)

Figure 2: (a) Ductal Echography (9 MHz), typical appearance of a lobe totally displayed from the nipple to the periphery of the breast. (b) Interpretation.

An anatomically guided method of investigation, based on the identification of the internal mammary structures, Ductal Echography (DE), was introduced in 1987 [2]. This technique is based on radial scanning, i.e. ductal-axial scans are performed along the ductal course. With this new technique, the observer actively investigates the epithelial structures by systematically following the ductal system in each mammary lobe. Figure 2 shows a DE and its interpretation. DE makes it possible for practitioners to visualize, delineate and hence to differentiate the lobe, Cooper's ligaments, the fasciae (superficialis and pectoralis), the fat tissue and the chest wall. Inside the lobe, sonographers can identify the ducts and the lobules when they are visible and quantify any changes in these structures in order to identify a mammary pathology. However, this technique has several drawbacks, such as the need for well-trained operators: (i) DE views of the breast are only partial, (ii) the difficulty of handling this method increases with the complexity of the lobular structures examined.

Our research aims at developing ultrasound tomography techniques [4] - [8] that will reduce the operator-dependency observed in echography and provide an intelligible visualization of the epithelium. Tomographic methods in medical ultrasound are the subject of increasing interest [9] - [12]. One of the main goals in this field of research is to improve the differentiation of breast tissues (and cancers) by quantifying the sound speed, the elasticity and the attenuation of tissues [9] - [19] and as an added benefit, to improve the reflected ultrasound images by using local sound speed and absorption maps [20] [21] [8]. We propose to differentiate the breast tissues using not only these parameters, but also 
the breast anatomy identification as performed in DE [2].

Several groups have attempted to understand the physical causes of ultrasonic wavefront distortion either by performing direct measurements on the human breast [22] [23] or by devising models of breast tissues. In one case, [24] simulations were conducted through a simple two-dimensional model of the breast in order to study the limitations of the Born approximation used in ultrasound imaging techniques. However, this model did not take into account the detailed structure of breast tissues. In another study [25], ultrasonic propagation through the abdominal wall was simulated using anatomically realistic tissue cross sections.

The aim of the present study was to develop 2-D anatomic breast computer phantoms that can be used to generate ultrasonic images. This 2-D breast model does not take into consideration the refraction and scattering effects that can occur in a real 3-D breast. However, in practical situations, due to technological limitations (such as the data acquisition and the cost), the most operational imaging systems are 2-D systems. Beamforming techniques are used to reduce the $3-\mathrm{D}$ scattering effects by taking a slice $2-3 \mathrm{~mm}$ in width as the region of interest (ROI). Consequently, we assume that 2-D computations are suitable means of simulating 3-D focused wave propagation through tissues.

In order to simulate ultrasonic propagation through the 2-D breast model, we use a finite element time domain method that has the advantage of requiring no physical approximations (within the framework of linear acoustics). Our method therefore automatically accounts for multiple scattering, refraction and reflection. The 2-D anatomic phantom proposed is an axial cross-section of the ductolobular structure in healthy and pathological situations. The various constitutive tissues are modeled as a random inhomogeneous continuum with density and sound speed fluctuations [26] - [29].

The goal is also, from the practitioner's qualitative point of view, to compare the recognized DE reference with the high-potential tomographic approach. Moreover, the present systems using ultrasound scattering tomography are capable of imaging cross sections of the breast in coronal format from the chest wall to the nipple and then of generating a 3 -D data set for the entire breast. The orientation of these systems (perpendicular to the ductal course) does not provide sufficient resolution and contrast to match the epithelial arborescence. A semi-circular antenna that would make it possible to image diametric sections of the breast passing through the nipple (Fig. 3) would be more appropriate for investigating the breast ductal tree and thus to reveal lesions at an early stage.

For DE, we simulate a linear probe, and a semi-circular antenna is used for DT. Qualitative comparisons were made between DT and DE on an anatomical basis.

\section{Wave propagation modeling}

The numerical method used to compute the solution of the wave equation was previously described in [31]. This method models the time domain acoustic wave propagation in fluid media and is based on the discretization of the mixed velocity-pressure formulation for acoustics. The space discretization of the problem is based on a mixed finite element 
method [32] and for the discretization in time, a 2nd order-centered finite difference scheme is used. The simulation grid is surrounded by an absorbing layer (PML) simulating unbounded domains [33].

This numerical method models the breast as a fluid of variable velocity and density. Experimental results obtained by other authors [34], [35] have shown that in soft tissues, the absorption of shear waves is much greater than the absorption of pressure waves. This enables us to adopt an acoustic model at the frequencies $(2-10 \mathrm{MHz})$ used in diagnostic ultrasound contexts.

The computational model used here does not account for the attenuation. Not accounting for attenuation was assumed not to affect the accuracy of the results, since with current echographic and tomographic imaging methods, the acoustic attenuation in the tissues is compensated for by applying a time-varying amplification during receiving. This time-varying amplification is referred to as Time Gain Compensation (TGC).

\section{Numerical phantom}

\subsection{Tissue random media}

Scattering in tissues arises from spatial changes in the acoustic impedance about the local mean value. The tissue model used here assume a sparse collection of randomly distributed scatterers. In the tissue model, a 2-D spatial autocorrelation acoustic impedance function can be used to describe the size, shape, distribution, and mechanical properties of the scatterers in the medium [26] - [28]. Some authors [26] have suggested calculating the acoustic intensity form factor. The form factor is proportional to the Fourier transform of the autocorrelation function and describes the frequency dependence of the scattering, based on the size, shape, and mechanical properties of the scatterers. The Gaussian form factor given by (1) has been used to model the scattering properties of many soft tissues [26] - [28] [30]. The Gaussian form factor represents a distribution of continuously changing impedance with the surrounding tissues and has an effective radius which characterizes the impedance distribution of the scatterers. A specific couple (correlation length, standard deviation) is thus assigned to each kind of tissue: it quantifies the fluctuation distributions (in terms of both the density and sound speed) around the mean values. We describe below how random media are generated.

Each parameter consists of a mean value to which we add the realization of a stationary (statistically homogeneous) random medium. The standard deviation of this realization is correlated with the mean value. The stationary random medium is expressed in terms of white noise filtered by a spectral filter (the Gaussian form factor). In this way, the medium covariance function can be expressed in terms of the correlation function [26] [29].

Each of the acoustic parameters (density and sound speed), noted $u$, is described by its spatial distribution $u=u(\mathbf{x})$, with a mean value $u_{0}(\mathbf{x})=\langle u(\mathbf{x})\rangle$. The difference $U(\mathbf{x})=u(\mathbf{x})-u_{0}(\mathbf{x})$ between the parameter and its mean value can be characterized by the correlation function $\mathrm{C}(\mathbf{x}, \mathbf{y})=\left\langle U\left(\mathbf{x}+\frac{1}{2} \mathbf{y}\right) \cdot U^{*}\left(\mathbf{x}-\frac{1}{2} \mathbf{y}\right)\right\rangle . \mathbf{x}$ is the midpoint of the pair of points $\mathbf{x}_{1}=\mathbf{x}+\frac{1}{2} \mathbf{y}, \mathbf{x}_{2}=\mathbf{x}-\frac{1}{2} \mathbf{y}$ where the correlation is observed, whereas $\mathbf{y}$ is the distance between the points. The correlation function depends only on $\mathbf{y}=\mathbf{x}_{1}-\mathbf{x}_{2}$ in the statistically homogeneous random medium approximation $\mathrm{C}(\mathbf{x}, \mathbf{y})=\mathrm{C}(\mathbf{y})$. The medium 
is constructed by performing a series of steps. First, a pseudo-random realization $\omega(\mathbf{x})$ of a white noise of unit standard deviation is generated on a regular rectangular grid. Next, we calculate the 2-D Fourier transform $\widetilde{\omega}(\mathbf{k})$ of this realization and multiply it by the spectral filter $\widetilde{F}(\mathbf{k}), \widetilde{U}(\mathbf{k})=\widetilde{F}(\mathbf{k}) \cdot \widetilde{\omega}(\mathbf{k})$. The 2-D inverse Fourier transform $U(\mathbf{x})$ of the product $\widetilde{U}(\mathbf{k})$ is then calculated. The mean value $u_{0}(\mathbf{x})$ is subsequently added to obtain the desired pseudo-random realization: $u(\mathbf{x})=u_{0}(\mathbf{x})+U(\mathbf{x})$. In consequence, the Fourier transform of the correlation function is the power spectrum of the filter $\widetilde{\mathrm{C}}(\mathbf{k})=\widetilde{F}(\mathbf{k}) \widetilde{F}^{*}(\mathbf{k})$. We now focus on isotropic homogeneous correlation functions of the type $\widetilde{F}(\mathbf{k})=\widetilde{f}(k)=\widetilde{f}(\|\mathbf{k}\|)$. We use the Gaussian spectral filter [26] - [28]

$$
\tilde{f}(k)=e^{-\frac{a^{2} k^{2}}{8}}, \quad a \in \mathbb{R}^{*},
$$

where $a$ is the Gaussian correlation length that represents the average radius of the scatterer. The Gaussian spectral filter therefore gives the correlation function:

$$
\mathrm{C}(\mathbf{y})=\frac{1}{\pi a^{2}} \cdot e^{-\frac{\mathbf{y}^{2}}{a^{2}}} .
$$

\subsection{Anatomic numerical phantom: a diametric section of the breast}

Figure 3 gives the sound speed and density maps (used for computation) of a diametric section of the breast passing through the nipple of a healthy subject (right lobe) and a pathological case of duct infiltration (ectasia: left lobe). The drawings (Fig. 3 and 4) show the successive layers of the breast successively probed by the ultrasound: the skin, the fat (including the superficial fatty layer and the deep fatty layer), the lobe, the connective tissue supporting the lobe and the intra-lobular structures. Cooper's ligaments link the lobes to the superficial layer of the superficial fascia underlying the skin and to the deep layer of the pectoralis fascia lining the thoracic wall [2].

Each pixel of the map is then assigned a sound speed and a density value, based on the results found in the relevant literature, which are assumed to be representative of the local tissue type. The water sound speed and density are those of water at $25{ }^{\circ} \mathrm{C}$. The skin and connective tissue sound speeds used were based on [14] and [17], respectively. Sound speeds for fat, glandular tissue and pectoralis muscle were obtained by the average values of human tissues given in [13] and [14]. Sound speeds for the ductolobular tree in the healthy and pathological cases were determined as described by [13] and [15]. The density values used for adipose tissue (or fat), mammary gland, skeletal muscle, and skin are average values from [36].

The standard deviation and the correlation length of each tissue are chosen in order to restitute (from the radiological point of view) a realistic echographic image. The correlation lengths (and standard deviations) chosen for the connective tissue (fasciae and Cooper's ligament), the hyper echogenic skin and the lobe are smaller (and higher, respectively) than those chosen for the pectoralis muscle. The correlation length of the duct infiltration equals the size of the average scatterer diameter of $30 \mu \mathrm{m}$ reported by [28]. In order to be consistent with the echographic rendering of the fatty tissue, and since fat is a rather homogeneous medium including few scatterers, we distribute the fat scatterers using a Bernouilli Gaussian distribution. The equivalent Bernouilli Gaussian distribution is deduced from a Gaussian law (a $2 \%$ standard deviation and a $0.2 \mathrm{~mm}$ correlation length) 
threshold of $90 \%$ of the standard deviation. The parameter values employed in the present study are summarized in Table 1.

Table 1: Reference values for acoustic properties

\begin{tabular}{|c|c|c|c|c|}
\hline \multirow[b]{2}{*}{ Medium } & \multicolumn{2}{|c|}{ Average } & \multicolumn{2}{|c|}{ Random medium } \\
\hline & $\begin{array}{l}\text { sound speed } \\
(\mathrm{m} / \mathrm{s})\end{array}$ & $\begin{array}{l}\text { Density } \\
\left(\mathrm{kg} / \mathrm{m}^{3}\right)\end{array}$ & $\begin{array}{c}\text { Standard } \\
\text { deviation } \\
(\%)\end{array}$ & $\begin{array}{l}\text { Correlation } \\
\text { length } \\
(\mathrm{mm})\end{array}$ \\
\hline Water & 1500 & 1000 & - & - \\
\hline Skin & $\begin{array}{c}1590 \\
{[14][17]}\end{array}$ & $\begin{array}{l}1090 \\
{[36]}\end{array}$ & 2 & 0.21 \\
\hline Connective & $\begin{array}{c}1545 \\
{[14][17]}\end{array}$ & $\begin{array}{c}1120 \\
{[36]}\end{array}$ & 2 & 0.21 \\
\hline Fat & $\begin{array}{c}1470 \\
{[13][14]}\end{array}$ & $\begin{array}{l}950 \\
{[36]}\end{array}$ & \multicolumn{2}{|c|}{ Bernouilli Gaussian } \\
\hline Lobe & $\begin{array}{c}1550 \\
{[13][14]}\end{array}$ & $\begin{array}{l}1060 \\
{[36]}\end{array}$ & 2 & 0.21 \\
\hline Muscle & $\begin{array}{c}1545 \\
{[13][14]}\end{array}$ & $\begin{array}{l}1050 \\
{[36]}\end{array}$ & 0.8 & 0.42 \\
\hline Ectasia & $\begin{array}{c}1570-1620 \\
{[13][15]}\end{array}$ & $\begin{array}{l}1040 \\
{[36]}\end{array}$ & 2 & $\begin{array}{l}0.03 \\
{[28]}\end{array}$ \\
\hline
\end{tabular}

The finite element code described in [section 2] was used to compute the propagation of cylindrical waves (2-D simulations) through the phantom. The spatial step size of the finite element grid was chosen to be one thirtieth of the wavelength. Each simulation was performed on IDRIS (Institute for Development and Resources in Intensive Scientific computing) using a Compaq Linux Cluster incorporating 24 Alpha EV68 processors cadenced at $836 \mathrm{MHz}$ (1.5 Gigaflop peak performance per processor).

\subsubsection{Numerical breast phantom for echography}

During the clinical examination, the patient is lying supine and her breast is flattened. A water bag is placed on the probe for coupling to the transducer, and thus the skin appears rectilinear. For the Finite Element Code, a grid of $4000 \times 2500$ pixels $(\Delta x=0.01 \mathrm{~mm}, 4$ $\mathrm{cm} \times 2.5 \mathrm{~cm}$ ) was used. Signals were recorded by 256 receivers simulating a linear probe placed about $0.9 \mathrm{~cm}$ from the skin surface. The computation time required to run the whole simulation was of the order of 2800 hours ( 256 transmitters $\times 11$ hours). Figure 4 gives the sound speed and density maps for an ectasia pathological situation.

\subsubsection{Numerical breast phantom for tomography}

During the ultrasound tomographic examination, the patient is lying prone on an examination bed, her breast immersed in a water bath and surrounded by a semi-circular antenna. For the Finite Element Code, a grid of $2400 \times 1200$ pixels $(\Delta x=0.045 \mathrm{~mm}$, $10.8 \mathrm{~cm} \times 5.4 \mathrm{~cm}$ ) was used (Fig. 3). Short pulses were transmitted from the transducers facing the reconstructed quadrant at every $0.25^{\circ}$. The computation time required to run the whole simulation was of the order of 720 hours ( 360 shots $\times 2$ hours). 

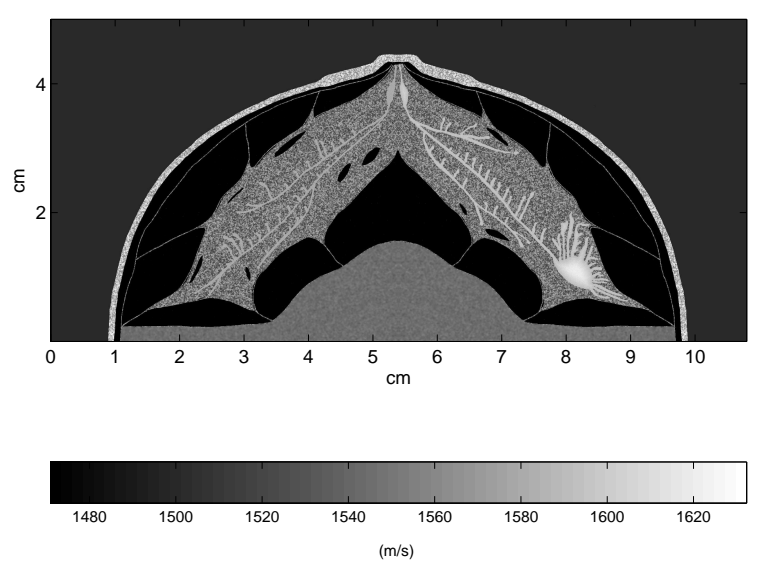

(a)

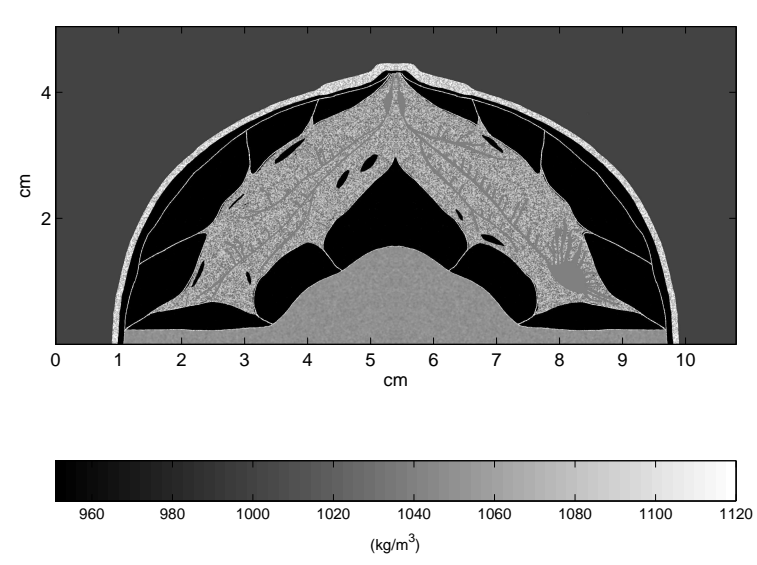

(b)

Figure 3: Numerical breast phantom for tomography. The patient is lying prone on an examination bed, her breast immersed in a water bath. (a) Sound speed map and (b) density map.
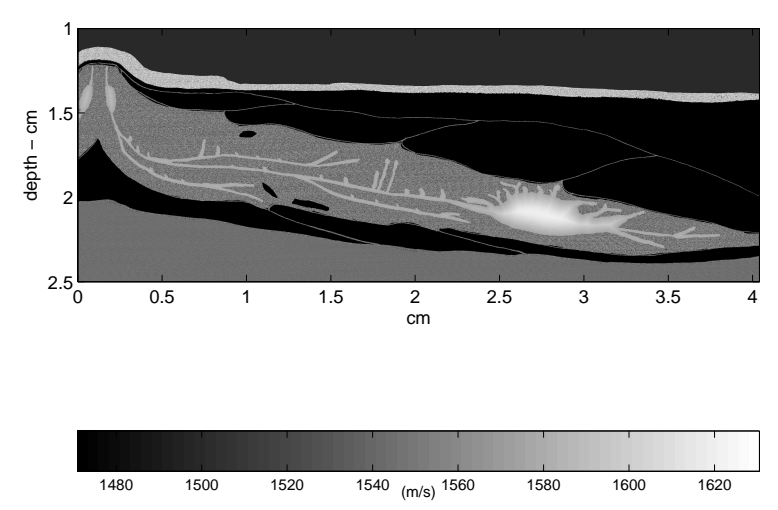

(a)
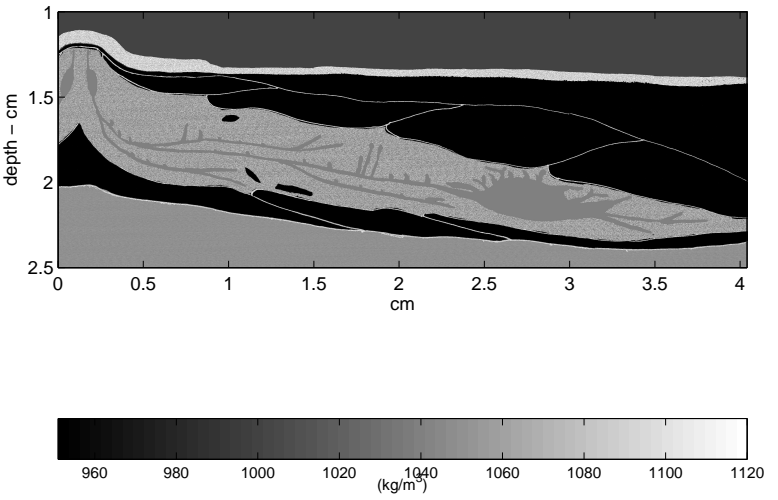

(b)

Figure 4: Numerical breast phantom for echography. The patient is lying supine and her breast is flattened. (a) Sound speed map and (b) density map. 


\section{Results}

A unifying theory that relates Synthetic Aperture (or Focusing) Imaging to back-projection reconstruction methods has been developed by several authors [37] - [39]. This unifying theory makes it possible to analyze active imaging systems in terms of ellipsoidal analysis (i.e. projections and back-projections on ellipsoidal surfaces), and provides direct insights into the predictable benefits of ellipsoidal backprojection tomography in comparison with conventional echography [39]. The EBP technique has the advantage of offering a high resolution power and is easy to implement [5] [8].

In order to show the progress achieved by tomographic reconstruction in comparison with the echographic technique, we voluntarily chose to operate at a frequency four times lower in tomography than in echography. In addition, these frequencies are suitable for a dual approach involving both reflection and transmission tomography.
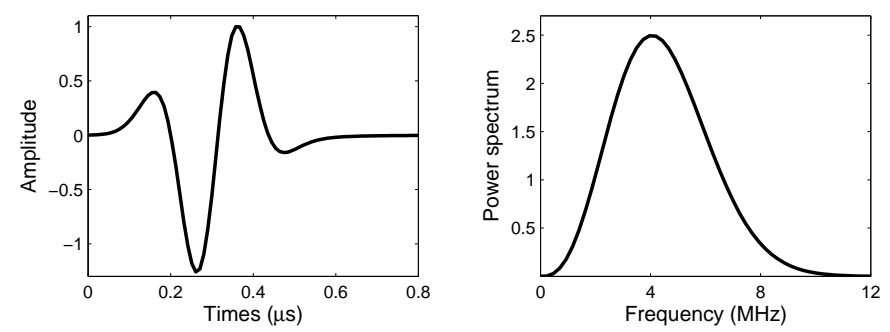

Figure 5: $4 \mathrm{MHz}$ cylindrical wave used in the FEM simulations in time and in frequency.

For echography, we simulate 256-element transducers with a central frequency of 4 $\mathrm{MHz}$. It is worth noting here that DE operates at higher frequencies (7 to $13 \mathrm{MHz}$ ). The use of lower frequency $(4 \mathrm{MHz})$ in our simulation is due to an acceptable computation time constraints [section 3.2.1]. The piezoelectric transducers are represented by punctual sources (used both in emission and reception), and are evenly spaced at a distance of $\frac{\lambda_{0}}{3}$. Each punctual source transmits a cylindrical wave (2-D simulation), the waveform $f(t)$ of which is defined as follows:

$$
\begin{gathered}
f(t)=4 \pi^{4} f_{0}^{4}\left(t-\frac{1}{f_{0}}\right)\left(3-2 \pi^{2} f_{0}^{2}\left(t-\frac{1}{f_{0}}\right)^{2}\right) \\
\cdot e^{-\pi^{2} f_{0}^{2}\left(t-\frac{1}{f_{0}}\right)^{2}} .
\end{gathered}
$$

The temporal and spectral plots of the transmitted pulses are shown in Fig. 5. An aperture with 32-active elements is employed. We use transmission and reception focusing (via beamforming) at $1.1 \mathrm{~cm}, 1.35 \mathrm{~cm}, 1.6 \mathrm{~cm}, 1.85 \mathrm{~cm}, 2.1 \mathrm{~cm}$ and $2.35 \mathrm{~cm}$, which gives a dynamic scanning range of $1 \mathrm{~cm}$ to $2.45 \mathrm{~cm}$. The reconstructed image (Fig. 6) is produced by assuming straight-line propagation, and a mean sound speed of $1540 \mathrm{~m} / \mathrm{s}$.

For tomography, point-like transducers with a central frequency of $1 \mathrm{MHz}$ move along a semicircle with a radius of $5 \mathrm{~cm}$. For each shot transmitted, numerical signals were recorded by means of point-like receivers over a finite aperture of $20^{\circ}$ centred on the transmitter, with a step of $0.25^{\circ}$. The waveform $f(t)$ given in (3) is used. The tomographic image (Fig. 7) is reconstructed using the EBP procedure presented in [5] [8]. 
The simulated echographic view (Fig. 6) can be compared with the phantom in Fig. 4. It can be seen here that the main features (lobe, ligaments, fat and retro-areolar zone) are correctly imaged. The DE image suffers from some typical echographic limitations. The retro-areolar zone, which is a sensitive area in cancer detection, is poorly depicted. The borders of the lesion, which are of great semiologic importance, are poorly imaged when they are parallel to the probe axis. In addition, the textures of the tissues are band-pass and spatially filtered (the point spread function of the echograph is frequency dependent and non isotropic).

The DT image (Fig. 7), on the contrary, shows an almost isotropic speckle. The various speckle distributions limit the lateral resolution of the DE images in comparison with DT ones. Here we can appreciate the presence of the fatty inclusions (which are not detected by the echograph) and the quality of the retro-areolar zone. The border of the lesion is delineated, although the center frequency of the tomographic signals is one quarter of that of the echographic pulses. In both cases, the ducts are not visible, since the impedance contrast is weak and their sizes smaller than the wavelength.

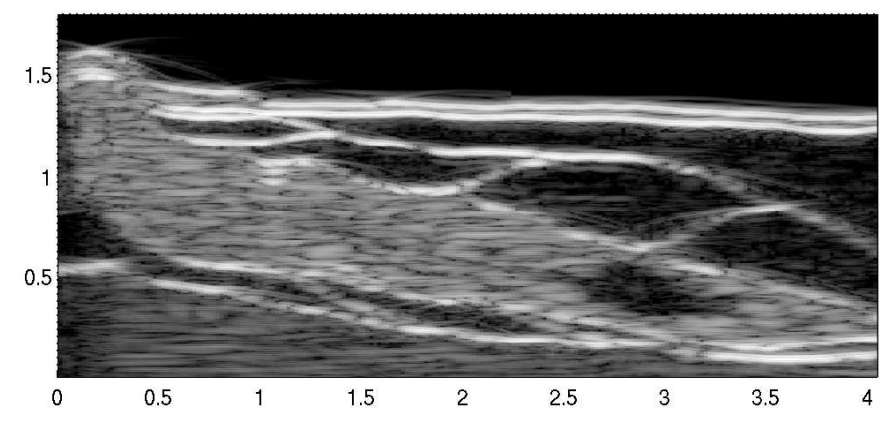

Figure 6: Ductal Echographic reconstruction (4 MHz), linear scanning.

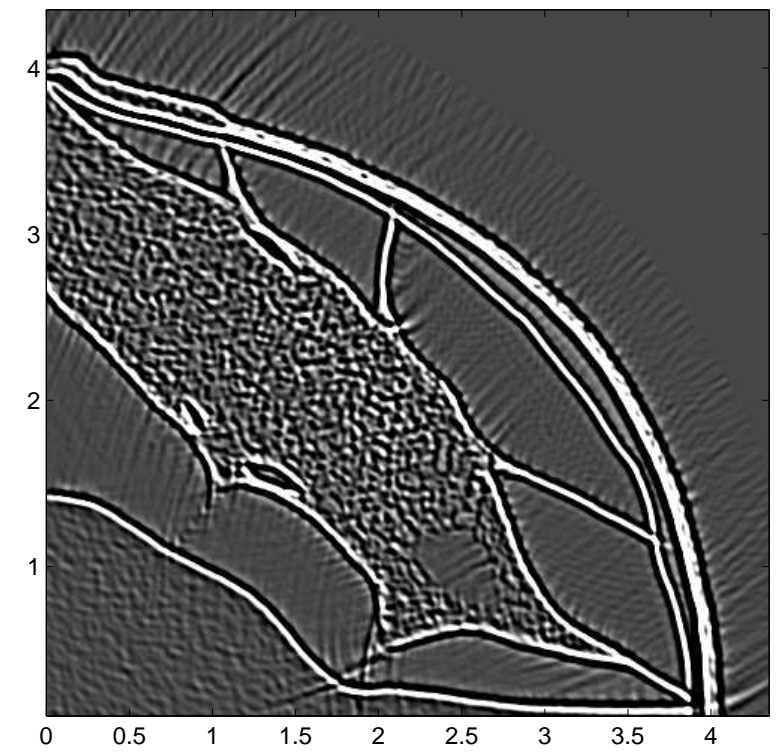

Figure 7: Ductal Tomographic reconstruction (1 MHz). 


\section{Discussion}

As often occurs with simulations of propagation through tissue [24] [25], the accuracy of the simulations through the 2-D anatomic breast phantoms described here is limited by the simplification of the real tissue structures. Although care was taken to ensure that the sound speed and density values were fairly representative, the tissue model is limited by the absence of microstructural information about the tissue. Neither histological information nor mechanical information was used, except for the duct infiltration, the correlation length of which is equal to the size of the average scatterer diameter of $30 \mu \mathrm{m}$ (the average scatterer diameter in the case of carcinomas given in [28]). A tomographic reconstruction analysis based on this model would therefore not be valid if one attempts to develop a tomographic-based microstructural description of the tissue. Likewise, the speckle resulting from the random media used to simulate the breast tissue may be different from that which would result from actual scatterers. The lack of information from which the model suffers has some undesirable side-effects. We are not able to quantitatively measure the accuracy of an ultrasound device from the end-user's point of view (that of the anatomopathologist or senologist). The detection performances of ultrasound systems are too low and the false negative rates too high. In short, it is difficult to determine what relevant the findings are and how to make the imaging system more accurate. This shows the need to carry out active research projects bridging the histological and acoustic domains.

Secondly, in a healthy subject, since the tissue attenuation is counterbalanced by the time gain compensation, omitting the attenuation processes seems to be justified. In pathological cases (where senologists need to assess the malignancy of the lesion), the attenuation process is of prime importance and the numerical model we propose is therefore not suitable. However, when the attenuation is largely due to scattering effects (in the case of a specular lesion, for example), the absence of absorption will probably have little effect on the results. Further studies will be required to confirm this assumption.

Consequently, the use of the numerical breast model described here should be limited to imaging and locating scatterers which are either coherently distributed (tissues interfaces) or incoherently spaced (textures).

Several other assumptions made in the 2-D breast model were not expected to significantly affect the results. The shear elasticity of the tissues was neglected in our computations because tissues have been found to be satisfactorily approximated by a fluid model at the frequencies $(2-10 \mathrm{MHz})$ used for diagnostic ultrasound purposes [34], [35].

The present method involved the use of two-dimensional computations, whereas real ultrasonic propagation occurs in three dimensions. This limitation is due to the fact that, at present, 3-D simulations are beyond the capacity of middle of the range computers (computation time). For an equivalent 3-D simulated tomographic experiment, a grid of $2400 \times 2400 \times 1200$ pixels $(\Delta x=0.045 \mathrm{~mm}, 10.8 \mathrm{~cm} \times 10.8 \mathrm{~cm} \times 5.4 \mathrm{~cm})$ would have to be used. The computation time required for one transmitter would be at least 4800 hours or 200 days $(2$ hours $\times 2400$ pixels). Although parallelization of the finite element code reduces the computation time, the computational resources available at present mean that our simulations have to be restricted to a 2-D model. Nevertheless since 2-D sections of tissue of real 3-D breast are imaged using a beam focused in the elevation direction (in the case of both the DE and DT approach), 2-D computations can be expected to accurately describe the wavefront distortion as the focused ultrasonic wave propagates 
through tissues.

Despite the limitations of the model presented here, the DE image (Fig. 6) matches current DE views (Fig. 2). This indicates that the finite element code method and the tissue model employed are sufficiently accurate to reproduce the main effects of the refraction and scattering, that have to be accounted for in overcoming reconstruction imaging issues.

\section{Conclusion}

A 2-D numerical acoustic model of the breast is presented here. The main features of this phantom are the fact that it accounts for anatomic details on the sub-millimeter scale as well as the ultrasonic characteristics of the various types of tissues constituting the breast (lobe, connective tissues, fat, and muscle). These characteristics are expressed in terms of density and celerity maps. However, accurate modeling would require three-dimensional computations, more refined tissue models (to reproduce the speckle) as well as a viscoacoustic extension of the finite element code.

This phantom was used as a computational tool to evaluate ultrasonic imaging reconstructions. This paper has shown that it is possible to generate realistic simulated ultrasound images. The 2-D breast phantom described here enables us to simulate DE images, using $4 \mathrm{MHz}$ linear array probes, that match current ultrasound DE views. The simulated echographic image obtained was compared with the tomographic one. In the latter case, $1 \mathrm{MHz}$ cylindrical waves were transmitted and measured by point transducers uniformly distributed on a semi-circular antenna. Qualitative comparisons between these images show that an improvement has been made in the tomographic reconstruction in terms of both the contrast and the resolution. We have limited our analysis to an qualitative anatomical assessment; further research could be led to elaborate advanced quantitative criteria of image quality.

It is hoped that this breast model will lead to a better understanding of the composition and the structure of the breast and their interaction with ultrasound [23] [24]. Improving our knowledge of these interactions will make it possible to design more efficient future equipment and to develop more powerful tissue characterization techniques.

In addition, tomography can open the way to quantitative imaging methods, i.e., to computer assisted diagnoses. This is the next challenge to be met in the field of ductal ultrasound computed tomography. This goal can be achieved, thanks to the development of realistic breast phantom designs and numerical experiments based on clinical data.

\section{Acknowledgments}

This study was supported by Ville de Marseille, Region Provence-Alpes-Côte d'Azur, CNRS-LMA, and an "Action Concertée Incitative" of the French Ministry of Research and Technology (2001-2004). The authors thank the Institute for Development and Resources in Intensive Scientific computing (IDRIS), where the computations were performed. We are grateful to Chrysoula Tsogka (researcher at CNRS) for putting her propagation code at our disposal and Veronique Bourrillon (ENSPM) for her participation in the echographic reconstruction developments. 


\section{References}

[1] D. Amy, Critères échographiques de bénignité, dans Echographie mammaire, De l'image à la thérapeutique, Collection d'imagerie radiologique, Edition Masson, 1998, pp. 23-31.

[2] M. Teboul, Practical ductal echography, guide to intelligent and intelligible ultrasonic imaging of the breast, editorial Medgen, 2004, pp. 15-98.

[3] H. Tulinius, O. Bjarnason, H. Sigvaldeson, Tumours in Iceland. 10. Malignant tumours of the female breast: a histological classification, laterality, survival and epidemiological considerations, Acta Pathol. Microbiol. Immunol. Scand., pp. 96-229, 1988 .

[4] S. Mensah, J.-P. Lefebvre, Enhanced Compressibility Tomography, IEEE Trans. Ultrason. Ferroelec. Freq. Contr. 44(66), pp. 1245-1252, 1997.

[5] S. Mensah, R. Ferriere, Near field diffraction tomography, Ultrason. Imaging 24, pp. 135-146, 2002.

[6] P. Lasaygues, D. Tanne, S. Mensah, J.-P. Lefebvre, Circular antenna for breast ultrasonic diffraction tomography, Ultrason. Imaging 24, pp. 177-189, 2002.

[7] R. Ferriere, S. Mensah, Weakly Inhomogeneous Media Tomography, Ultrason. Imaging 25, pp. 122-133, 2003.

[8] S. Mensah, R. Ferriere, Diffraction tomography: a geometrical distortion free procedure, Ultrasonics 42, pp. 677-682, 2004.

[9] M. P. André, H. S. Janée, P. J. Martin, G. P. Otto, B. A. Spivey, D. A. Palmer, High-speed data acquisition in a diffraction tomography system employing large-scale toroidal arrays, International Journal of Imaging Systems and Technology, vol. 8, pp. 137-147, 1997.

[10] R. Stotzka, J. Wrfel, T. Mller, Medical imaging by ultrasound computer tomography, in Proc SPIE's International Symposium Medical Imaging, 2002, pp. 110-119.

[11] M. Ashfaq, H. Ermert, Ultrasound spiral computed tomography for differential diagnosis of breast using conventional ultrasound system, in Proc. of the 27th International Symposium Acoustical Imaging 27, 2004, pp. 627-633.

[12] J. W. Wiskin, D. T. Borup, S. A. Johnson, M. J. Berggren, T. Abbott, Full wave non-linear inverse scattering: high resolution quantitative breast tissue tomography, in Proc. of the 28th International Symposium Acoustical Imaging 28, 2005, to be published.

[13] G. Kossoff, E. K. Fry, J. Jellins, Average velocity of ultrasound in the human female breast, J. Acoust. Soc. Am. 53(6), pp. 1730-1736, 1973.

[14] S. A. Goss, R. L. Johnston, F. Dunn, Comprehensive compilation of empirical ultrasonic properties of mammalian tissues, J. Acoust. Soc. Am. 64(2), pp. 423-457, 1978. 
[15] G. H. Glover, Characterization of in vivo breast tissue by ultrasound time of flight computed tomography, Ultrasonic tissue characterization II, M. Linzer, ed., National Bureau of Standards Spec. Pub. 525, pp. 221-225, 1979.

[16] J. F. Greenleaf, R. C. Bahn, Clinical imaging with transmissive ultrasonic computerized tomography, IEEE Trans. Biomed. Eng., vol. BME-28, no. 2, pp. 177-185, 1981.

[17] F. S. Foster, M. Strban, G. Austin, The ultrasound macroscope: initial studies of breast tissue, Ultrason. Imaging 6, pp. 243-261, 1984.

[18] K. Richter, Clinical Amplitude/Velocity Reconstructive Imaging (CARI) - A new sonographic method for detecting breast lesions, Brit. J. Radiol., vol. 68, pp. 375-384, 1995.

[19] T. A. Krouskop, T. M. Wheeler, F. Kallel, B. S. Garra, T. Hall, Elastic moduli of breast and prostate tissues under compression, Ultrason. Imaging 20, pp. 260-274, 1998.

[20] J. Kim, S. B. Park, S. A. Johnson, Tomographic imaging of ultrasonic reflectivity with correction for acoustic speed variations, Ultrason. Imaging 6, pp. 304-312, 1984.

[21] T. Mller, T. Deck, L. Ming, R. Stotzka, D. Hpfel., Ultrasound-computertomography: image reconstruction using local absorption and sound speed profiles, presented at the 7th conference of the European Society for Engineering and Medicine, 2003.

[22] P. D. Freiburger, D. C. Sullivan, B. H. LeBlanc, S. W. Smith, G. E. Trahey, Two dimensional ultrasonic beam in the breast: In vivo measurements and effects, Ultrason. Imaging 14, pp. 276-299, 1992.

[23] L. M. Hinkelman, D.-L. Liu, R. C. Waag, Q. Zhu, B. D. Steinberg, Measurement and correction of ultrasonic pulse distortion produced by the human breast, J. Acoust. Soc. Am. 97(3), pp. 1958-1969, 1995.

[24] C. W. Manry, Jr. and S. L. Broschat, FDTD simulations for ultrasound propagation in a 2-D breast model, Ultrason. Imaging 18, pp. 25-34, 1996.

[25] T. D. Mast, L. M. Hinkelman, M. J. Orr, V. W.Sparrow, R. C. Waag, Simulation of ultrasonic pulse propagation through the abdominal wall, J. Acoust. Soc. Am. 102(2), pp. 1177-1190, 1997.

[26] M. F. Insana, R. F. Wagner, D. G. Brown, T. J. Hall, Describing small-scale structure in random media using pulse-echo ultrasound, J. Acoust. Soc. Am. 87(1), pp. 179-192, 1990.

[27] M. L. Oelze, J. F. Zachary, W. O. O'Brien, Characterization of tissue microstructure using ultrasonic backscatter: Theory and technique for optimization using a Gaussian form factor, J. Acoust. Soc. Am. 112(3), pp. 1202-1211, 2002.

[28] M. L. Oelze, W. O. O'Brien, Differenciation and characterization of rat mammary fibroadenomas and $4 T 1$ mouse carcinomas using quantitative ultrasound imaging, IEEE Trans. medical imaging, vol. 23, no.6, pp.764-771, 2004. 
[29] L. Klimes, Correlation functions of random media, Pure and Applied Geophysics, 159, pp. 1811-1831, 2002.

[30] F. L. Lizzi, M. Ostromogilsky, E. J. Feleppa, M. C. Rorke, M. M. Yaremko, Relationship of ultrasonic spectral parameters to features of tissue microstructure, IEEE Trans. Ultrason. Ferroelec. Freq. Contr. UFFC-34, no 3, pp. 319-329, 1987.

[31] C. Tsogka, Modélisation mathématique et numérique de la propagation des ondes élastiques tridimensionnelles dans des matériaux fissurés, $\mathrm{PhD}$ thesis, Université Paris Dauphine - Paris IX, December 1999.

[32] E. Bécache, P. Joly, C. Tsogka, An analysis of new mixed finite elements for the approximation of wave propagation problems, SIAM J. Numer. Anal. Vol.37, no.4, pp. 1053-1084, 2000.

[33] F. Collino, C. Tsogka, Application of the pml absorbing layer model to the linear elastodynamic problem in anisotropic heteregeous media, Geogysics 66, pp. 294-305, 2001.

[34] L. A. Frizzell, E. L. Carstensen, Shear properties of mammalian tissues at low megahertz frequencies, J. Acoust. Soc. Am. 60(6), pp. 1409-1411, 1976.

[35] E. L. Madsen, H. J. Sathoff, Ultrasonic shear wave properties of soft tissues and tissuelike materials, J. Acoust. Soc. Am. 74(5), pp. 1346-1355, 1983.

[36] H. Q. Woodard, D. R. White, The composition of body tissues, The British Journal of Radiology, 59, pp. 1209-1219, 1986.

[37] S. J. Norton, M. Linzer, Ultrasonic reflectivity tomography: reconstruction with circular transducer arrays, Ultrason. Imaging 1, pp. 154-184, 1979.

[38] F. Anderson, Active imaging Green's function, in Proc. of the 19th International Symposium Acoustical Imaging 19, 1993.

[39] F. Anderson, F. Morgan, Active imaging analysis via ellipsoidal projections, in Proc. of the 21th International Symposium Acoustical Imaging 21, 1995.

[40] G. Beylkin, Discrete Radon transform, IEEE ASSP 5, pp.162-172, 1987.

[41] G. Beylkin, R. Burridge, Linearize Inverse Scattering Problems in Acoustics and Elasticity, Wave motion 12, Elsevier Science, 1990. 